LBL- -29175

DE90 015635

Study of Hot Carrier Relaxation in Quantum Wells by Subpicosecond Raman Scattering

D.-S. Kim and P.Y. Yu

Department of Physics

University of California

and

Materials and Chemical Sciences Division

Lawrence Berkeley Laboratory

University of California

Berkeley, CA 94720

This work was suppurted by the Director, Olfice of Energy Research,

Office of Basic Enetgy Science, Materials Science Division, of the U.S. Department of Energy under Contreet No. DE-AC03-76SF00098. 


\title{
Study of Hot Carrier Relaxation in Quantum Wells By Subpicosecond Raman Scattering
}

\author{
Dai-sik Kim and Peter Y. Yu
}

\author{
Department of Physics. University of California, Berkeley, CA 94720 \\ and \\ Materials and Chemical Sciences Division \\ Lawrence Berkeley Laboratory \\ 1 Cyclotron Road, Berkeley, CA 94720
}

\begin{abstract}
Relaxation of hot carriers excited by subpicosecond laser pulses has been studied by Raman scattering in GaAs/AlAs multiple quantum wells with well widths varying between 100 and $1000 \mathrm{~A}$. The hot phonon population observed by Raman scattering is found to decrease with the well width despite the fact that the hot electron temperature remains constant. The results are explained in terms of coufinement of both electrons and optical phonons in quantum wells.
\end{abstract}

\section{INTRODUCTION}

Hot carrier relaxation in both bulk semiconductors and semiconductor microstructures has been of great interest lately. Various optical techniques have been used tr study these fast relaxation mechanisms especiall, with the aid of picosecond (ps) and femiosecond (fs) lasers. ${ }^{1-8}$ Among these optical techniques, Raman scattering is unique for studying hot carrier relaxation because it can provide information on both electrons and phonons at the same time. For example, using subpicosecond Raman scattering, $\mathrm{Kim}$ and $\mathrm{Yu}^{1}$ have recently shown that intervalley scattering is the dominant cooling mechanism of not electrons in bulk III- $V$ direct band gap semiconductors in subpicosecond regime. In this time scale, longitudinal optical (LO) phonons play only a minor role in hot electron cooling.

It has been shown experimentally and by theoretical calculations that both electrons and optical phonons are confined in GaAs/AlAs quantum wells. ${ }^{9-11}$ This confinement in general leads to modifications of the electron-phonon coupling and thus changes in the cooling mechanisms. ${ }^{2.12}$ In the experiment reported in this paper, electron and LO phonon temperatures are measured as functions of both electron density and quantum well width by subpicosecond time-resolved Raman scattering. We found that LO phonon temperatures were greatly reduced as the well thickness decreased whereas the electron temperature was almost the same as the bulk value. The latter result is consistent with conclusions of our previous work ${ }^{1,13}$ that in bulk GaAs and related semiconductors the cooling of hot electrons in subpicosecond time scale is mainly determined by the intervalley scattering of high energy electrons and not by intravalley electron-phonon scattering. We have considered various explanations for the decrease of hot phonon generation rate with well thickness. One important factor is that the wavevector of the LO phonon probed by Raman scattering varies with well thickness $L$ as $1 / L$. Another important factor is that most of the hot phonons generated in the intra- $\Gamma$-valley electron-LO phonon (Fröhlich) scattering cannot be probed by our experiment due to energy and momentum conservation in the quantum well plane during the Raman process. Based on these considerations, we conclude that intra- $\Gamma$-valley scattering cannot account for the small, but non-zero, hot LO phonon population we observe in quantum wells with $L=120$ and $230 \AA$. A possible source of the hot $L O$ phonons in these quantum wells may be the relaxation of hot electrons within the $L$ valleys. 


\section{EXPERIMENT AND RESULTS}

Our experiment was perfomed on GaAs/AlAs multiple quantum well samples grown on [00I] oriented GaAs substrate by molecular beam epitaxy (MBE). The (GaAs thickness/AlAs thickness) of these quantum wells are nominally $(120 / 120),(230 / 230),(500 / 500)$ and $(1000 / 1000)$ $\dot{A}$. The total thickness of the GaAs layers in all samples is around $3000 \AA$ to absorb most of the incident photons. Hot electrons were excited by subpicosecond pulses from a CPM laser operating at $2.0 \mathrm{eV}$ and with an energy of $0.3 \mathrm{~nJ}$ per pulse. The linewidth of the laser was reduced to $25 \mathrm{~cm}^{-1}$ by a one-plate birefringent filter inside the cavity. The autocorrelation traces of these pulses are consistent with pulses with a $\operatorname{sech}^{2}$ profile and full-width-at-halfmaximum (FWHM) of 600 fs. The samples were maintained at $77 \mathrm{~K}$ during the experiment.

The light scattering experiment was performed in a backscattering geometry with both the incident and scattered light polarized along the [ II 0 ] direction. The scattered radiation was analyzed with a standard double monochromator and detected with a cooled photomultiplier tube with photon counting electronics. The Raman spectra were superimposed on a luminescence background caused by recombination of thermalized electron-hole pairs. The photoexcited electron densities were calculated from the numter of photons absorbed and also determined by analyzing the luminescence lineshape. A typical luminescence spectrum is shown in Fig.1.

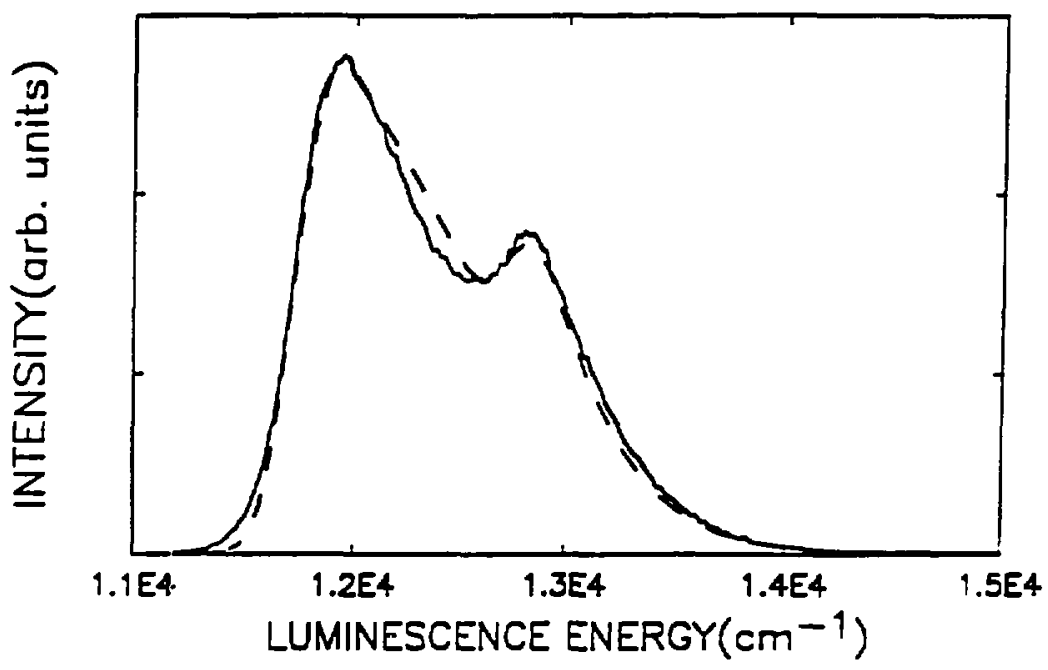

Fig. I The time-integrated photoluminescence spectrum for the $L=120$ A sample. The two peaks correspond to transitions from the first and second confined electronic levels to the firs: and second confined hole levels respectively. The broken curve is a theoretical fit based on a model of two-dimensional electron and hole plasma each in quasi-thermal equilibrium. The areal electron and hole densities deduced from this fit are $8.4 \times 10^{12} \mathrm{~cm}^{-2}$ corresponding to a bulk density of $7 \times 10^{18} \mathrm{~cm}^{-3}$. The sample temperature was $300 \mathrm{~K}$. 
This spectrum has been obtained from the $120 \mathrm{~A}$ sample at high excitation density. Two peaks are clearly visible and they correspond to recombination of electrons with holes from the first and second confined levels. The areal density determined from the luminescence spectrum is about $8.4 \times 10^{12} \mathrm{~cm}^{-2}$. A typical Raman spectrum of this sample obtained after subtracting the luminescence background is shown in Fig.2.

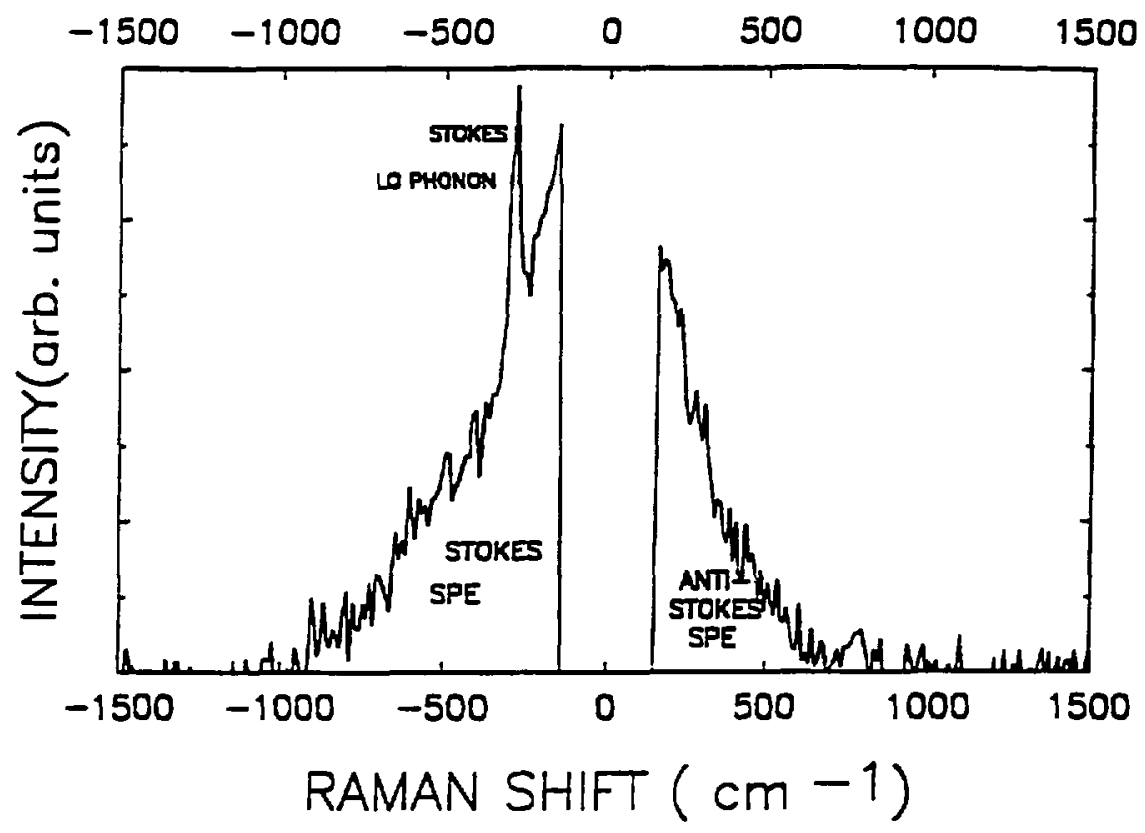

Fig. 2 A typical Raman spectrum of the $L=120$ A sample after subtraction of the luminescence background. The sharp peak at $-300 \mathrm{~cm}^{-1}$ is identified with Stokes-scattering of light by LO phonons. The anti-Stokes peak is barely visible because the phonon population is too low. The broader peak. on which the phonon is superimposed, is caused by scattering by SPE. The areal electron density for this spectrum is around $3.6 \times 10^{12} \mathrm{~cm}^{-2}$.

The Raman spectrum can be decomposed into relatively sharp peaks which correspond to Stokes and anti-Stokes phonon scattering, superimposed on a broader background assigned to scattering by single particle excitations (SPE) of hot electrons. Let us define $\mathrm{N}_{\mathrm{ph}}$ to be the occupation number of the phonon mode that is observed by our experiment. Let $T_{p b}$ be the phonon temperature defined as $N_{p h}=\left\{\exp \left(E_{L O} / k_{b} T_{p h}\right)-1\right\}^{-1}$ where $E_{L O}$ is the phonon energy, $k_{b}$ is Boltzmann's constant. Phonon occupation number is determined by comparing the Stokes and anti-Stokes intensities. ${ }^{14,15}$ In Fig.2, the anti-Stokes phonon peak is nearly invisible indicating a small phonon occupation number and thus low phonon temperature. Electron 
temperature $T_{\text {}}$ is deduced also from the ratio of Stokes to anti-Stokes intensities. ${ }^{1,16,17}$ Some of the measured values of $T_{e}$ and $T_{p h}$ are plotted against the well thickness $L$ in Fig. 3 . In obtaining these results, the three-dimensional electron density has been maintained at a constant value of $3 \times 10^{18} \mathrm{~cm}^{-3}$ by adjusting the laser focal spot on the sample.

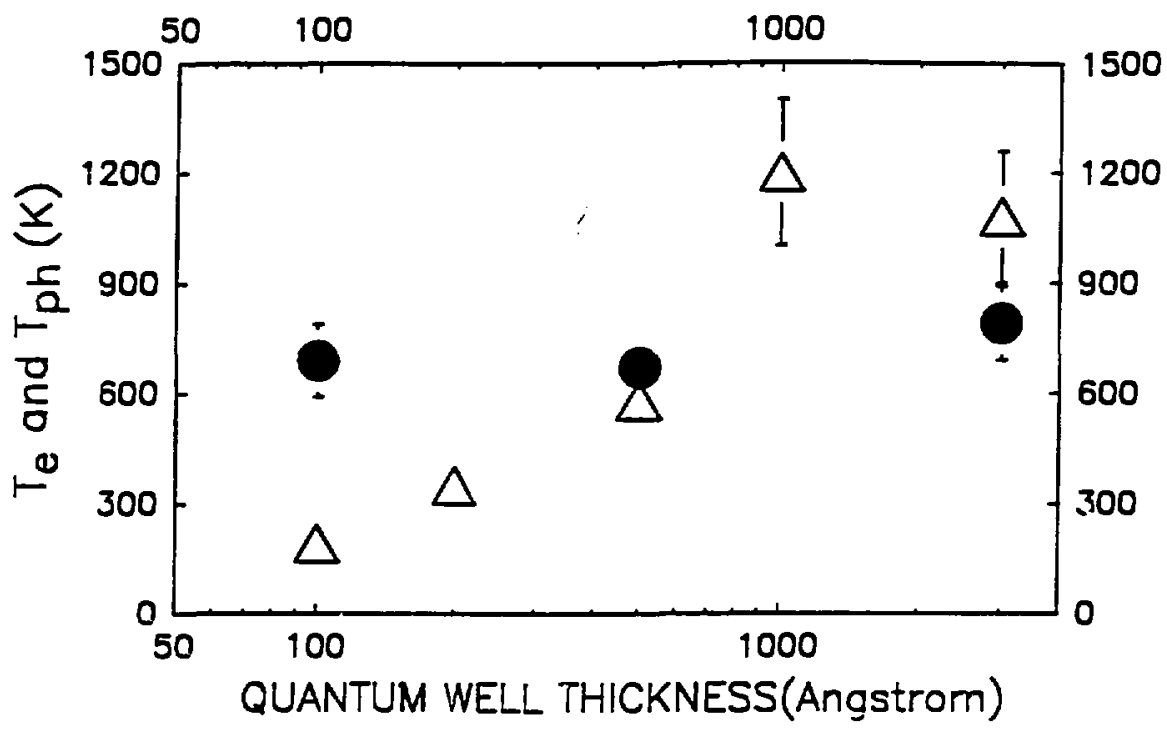

Fig. 3 The measured electron temperature $T_{e}$ (closed circles) and phonon temperature $T_{p h}$ (open triangles) plotted as functions of wall thickness. The bulk density of the electrons in all samples was kept a constant value of around $3 \times 10^{18} \mathrm{~cm}^{-3}$.

From Fig.3, we see that the electron temperature is almost independent of the well thickness whereas the phonon temperature increases with increasing thickness. Samples with $L>1000$ A behave essentially like bulk samples in that the phonon temperatures overshoot the electron temperature. The sample with $\mathrm{L}=3000 \mathrm{~A}$ has only a single well and can be considered really as a bulk sample. Electron temperatures are around $700 \mathrm{~K}$, as is the case of bulk GaAs. ${ }^{1,8}$ In bulk GaAs we have found that the hot phonon temperature increased rapidly with electron density $\left(n_{e}\right)$ for $n_{e}>10^{17} \mathrm{~cm}^{-3}$. At $n_{e}>10^{18} \mathrm{~cm}^{-3}$, the phonon temperature saturated at a temperature above $T_{e}$. We found that in the quantum wells the phonon population also increased linearly with $\mathrm{n}_{e}$ for $\mathrm{n}_{e}<10^{18} \mathrm{~cm}^{-3}$. We therefore defined a phonon generation efficiency as $\mathrm{dN}_{\mathrm{ph}} / \mathrm{dn}$ in the low density limit. This phonon generation efficiency is plotted as a function of well thickness in Fig. 4. We note that there is a decrease of almost 2 orders of magnitude in the phonon generation efficiency as we go from bulk GaAs to quantum wells with $\mathrm{L}=120 \mathrm{~A}$. 


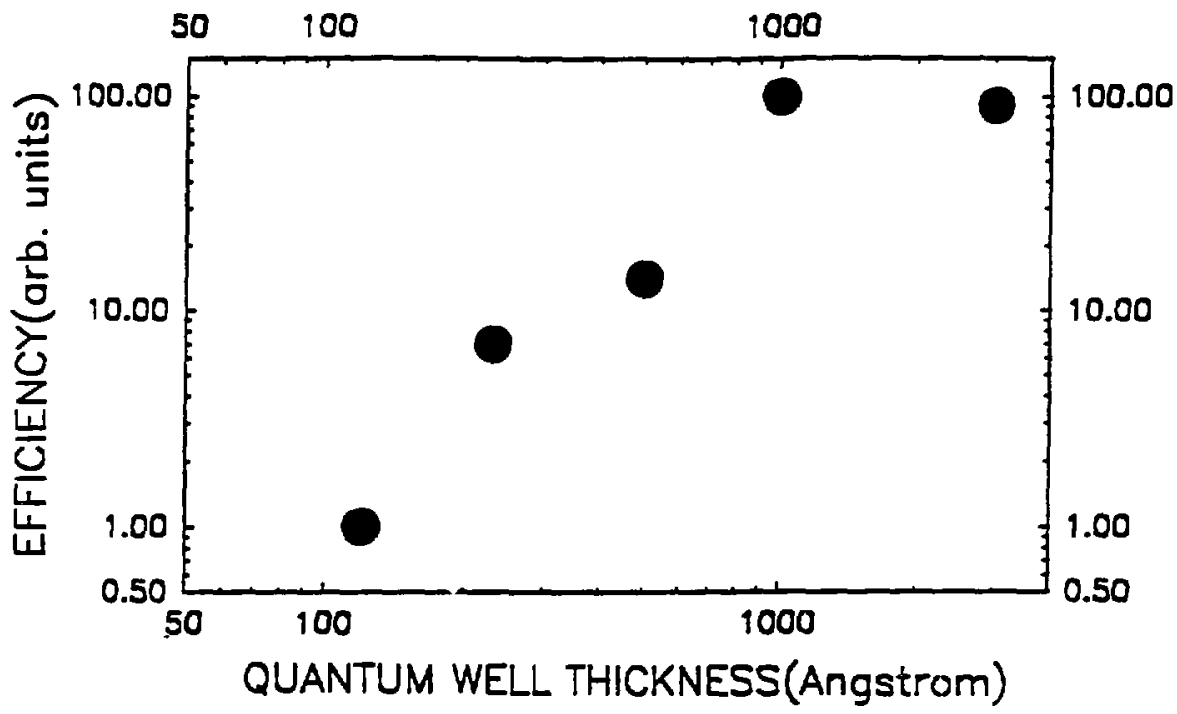
Fig. 4 Phonon generation efficiency $\left(\mathrm{dN}_{\mathrm{ph}} / \mathrm{dn}_{\text {}}\right.$ ) obtained from $\mathrm{N}_{\mathrm{ph}}$ vs $\mathrm{n}$, plots at low electron
densities $\left(<10^{18} \mathrm{~cm}^{-3}\right)$ as a function of quantum well thickness.

\section{DISCUSSIONS}

Confinement of phonons in GaAs quantum wells leads to quantization of phonon wavevectors along the growth direction of the sample. ${ }^{9-11}$ Let us define $q$ to be the wavevector of a phonon mode. In GaAs/AlAs multiple quantum wells with L>>lattice constant, the conf inement of optical phonons is almost perfect. We can decompose $q$ into two components: $q$ $=\left(Q, q_{2}\right)=(Q, m \pi / L)(m=1,2,3 \ldots)$ where $Q$ is the component of the phonon wavevector along the well plane and $z$ is the growth direction.

When the well width is around $100 \mathrm{~A}$, this confinement of the LO phonons is expected to significantly affect the intra- $\Gamma$-valley electron-phonon scattering because $\pi / \mathrm{L}\left(\approx 3 \times 10^{6} \mathrm{~cm}^{-1}\right)$ is comparable in magnitude to the wavevector of the majority of phonons involved in intravalley Fröhlich interaction. On the other hand, it is reasonable to assume that intervalley scatterings are not going to be changed very much by confinement because zone edge phonons with wavevector $>>\pi / L$ are responsible for these processes. Furthermore, electrons that have sufficient energy to undergo intervalley scattering will be less affected by the barriers. Thus in subpicosecond time scale where hot electron relaxation is mainly determined by intervalley scatterings, we expect that the cooling of hot electrons in quantum wells will not 
differ significantly from bulk GaAs provided the well widths are bigger than $100 \AA$. This is precisely what we observe in our experiment. The electron temperature stays constant around $700 \mathrm{~K}$ as we vary the well thickness. Thus, we conclude that the intervalley scattering rates in quantum wells of the order of $100 \AA$ or larger are essentially the same as that of bulk GaAs.

To understand our data on the L.O phonons, we need to know the wavevector of the phonon modes probed in our Raman scattering experiment. Let us define $q_{0}=\left(Q_{0}, q_{20}\right)$ to be the wavevector of the phonons observed in Raman scattering. It is known that away from resonance, Raman scattering measures mainly phonon mode with $m=1 .{ }^{10}$ Thus, $q_{20}$ is equal to $\pi / L, Q_{0}$ is determined by the momentum transfer of photons in the $x y-p l a n e$ in our experiment. This is because in Raman scattering in quantum wells, components of momenta along the plane of quantum wells must be conserved. In our backscattering geometry, $\mathbf{Q}_{0}$ is very small, around $0.5 \times 10^{5} \mathrm{~cm}^{-1}$. In other words, the phonon mode we observe in our experiment has very small (almost negligible for our purpose) wavevector in the plane of quantum wells. The wavevector along the $z$ direction changes with well thickness as $\pi / L$. Thus the magnitude of wavevectors of the phonons we observe is larger than that of the phonon we observe in bulk sample (which is around $8 \times 10^{5} \mathrm{~cm}^{-1}$ ) when $L$ is bigger than say $400 A$. This has an important effect on the generation rate of phonons because the phonon generation efficiency by Fröhlich interaction is proportional to $1 / q^{3} .1,14$ Thus from this consideration alone, we expect a decrease by a factor of $\approx 60$ in phonon generation rate compared with the bulk when we decrease the well width to $120 \AA$.

There is another factor that further reduces the generation rate of phonons with wavevector $Q_{0}$ in the $x y$-plane. This is related to the momentum conservation along the $x y$ plane in the electron-phonon scattering. It arises from the fact that electrons and phonons are free to move along the well plane. To generate hot phonons that we observe with such small wavevector along the plane, change in the electron momentum along the plane has to be very small also in the scattering process. As shown by the schematic scattering diagram in Fig. 5 , phonons generated by intravalley electron-LO phonon scattering have wavevectors in the $x y$ plane typically bigger than some minimal values $Q_{A}$ and $Q_{B}$ for intrasubband and intersubband scattering respectively. For well width of the order of $100 \mathrm{~A}$, both $Q_{A}$ and $Q_{B}$ are larger than $5 \times 10^{5} \mathrm{~cm}^{-1}$. Since the transverse momentum $Q_{0}$ probed by Raman scattering is about ten times smaller, we do not expect to see any hot phonons at all in quantum wells with widths of the order of $100 \mathrm{~A}$. One possible exception is the case when the intersubband energy difference $E_{2}-E_{1}$ is very close to $E_{L O}$. In this special case, an electron can scatter from the second subband to the first subband by emitting an LO phonon with essentially zero $Q_{0}$. Thus it is necessary to consider alternate mechanisms to account for the hot phonons we observe in our samples with $L=120$ and $230 \AA$.

\section{ALTERNATE MECHANISMS OF HOT PHONON CREATION IN QUANTUMI WELLS}

The above analysis shows that the hot phonon generation efficiency measured by Raman scattering in quantum wells with $L=120$ and $230 \AA$ should be reduced by more than. 3 factor of 100 compared to the bulk GaAs with comparable plasma densities and temperatures. Therefore we should not see any hot phonons at all in our samples with $L=120$ and $230 \AA$. Yet we still observe some hot phonons in these samples as shown in Fig. 3. In bulk GaAs samples. the hot phonons measured in Raman scattering are produced mostly from intra- $\Gamma$-valley electron-LO phonon scattering. However, within one ps as much as half of the electrons excited 


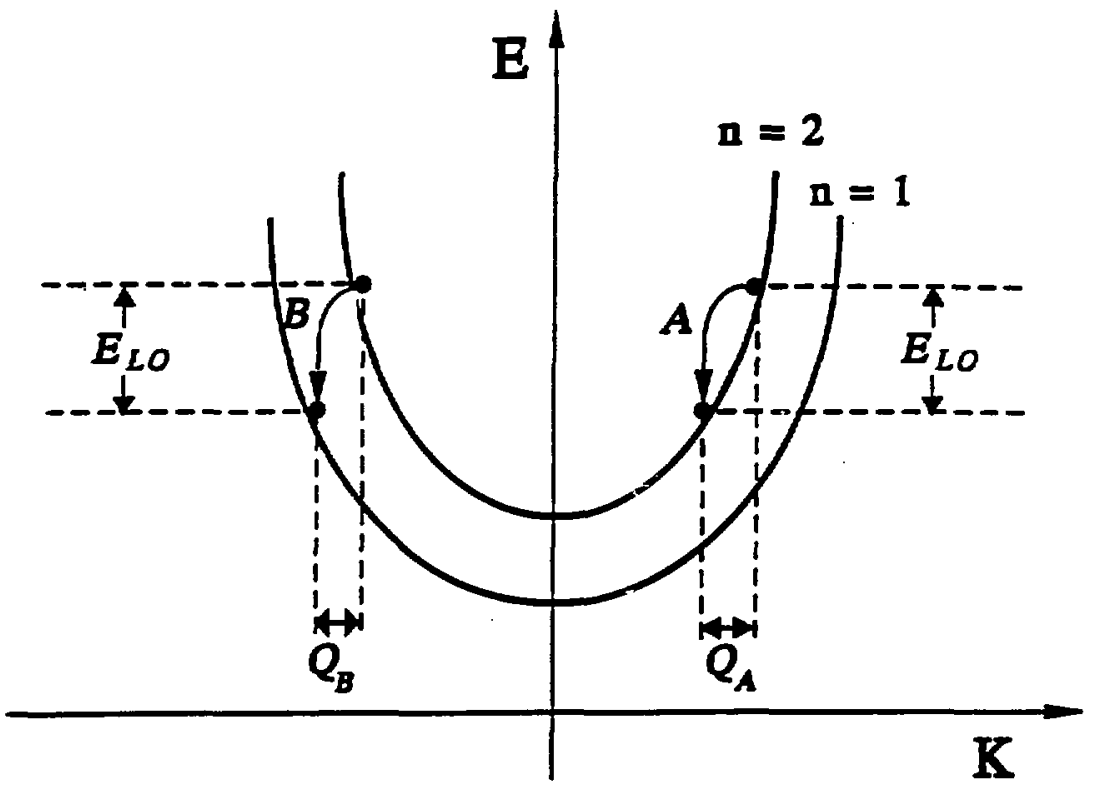

Fig. 5 A schematic diagram showing energy and momentum conservation in $x y$-plane in electron-LO phonon scattering. In $A$ (intrasubband) and $B$ (intersubband) processes, the smallest $Q$ of $L O$ phonons that can scatter the electron are denoted by $Q_{A}$ and $Q_{B}$ respectively.

by the laser pulses are scattered into the $L$ and $X$ valleys. ${ }^{1,8}$ Return of these electrons back to $\Gamma$ valley takes much longer, typically a few ps." Once the electrons are in these satellite valleys, they will relax by intravalley or intervalley scatterings. Zone edge phonons will be involved in the intervalley scatterings while smaller wavevector ( typically $>10^{6} \mathrm{~cm}^{-1}$ ) LO phonon will be generated by intravalley scatterings. The hot $L O$ phonons generated by intra- $L$ and intra-Xvalley scatterings normally have wavevectors too large to be observed by Raman scattering in bulk GaAs samples. In quantum wells, however, the wavevector of phonons observable in Raman scattering can be as large as $10^{6} \mathrm{~cm}^{-1}$ depending on $L$. These arguments are not affected by possible confinement of electrons in the satellite valleys. Such confinement effect is not expected to be significant because electrons in the $L$ and $X$ valleys have bigger effective masses than that of the $\Gamma$ valley electrons and also because the barrier heights are lower for electrons in the $\mathrm{L}$ and $\mathrm{X}$ valleys. Thus, relaxation of hot electrons in $\mathrm{L}$ and $\mathrm{X}$ valleys either by intra- or intervalley scatterings in GaAs/AlAs quantum wells should be essentially same as in 
bulk GaAs. Thus the emission of hot phonons during the intravalley relaxation of hot electrons in the higher conduction band valleys is a possible source of hot phonons observed in our $\mathbf{L}=120$ and $230 \AA$ quantum well samples. We have performed a preliminary calculation of the hot LO phonon population generated in this way. In the model we have assumed that all the hot $L O$ phonons are produced in the $\mathrm{L}$ valleys. This assumption is reasonable because electrons in the $X$ valleys can relax to the $L$ valleys by emission of a zone-edge phonon. Although the deformation potential for this $X$ to $L$ intervalley scattering is not known with precision, based on the large density of available final states in the $L$ valleys we expect that the scattering time will be competitive with the intravalley relaxation time. A comparison between this calculation and our data suggests that the observed hot LO phonon population in the narrower quantum wells can be accounted for, provided the time it takes for a $\mathrm{L}$ valley electron to emit a $\mathrm{LO}$ phonon is around $70 \mathrm{fs}$. This intravalley scattering time is about three times shorter than that of an electron in the $\Gamma$ valley. Considering the larger effective mass of the $\mathrm{L}$ valley electrons, this shorter scattering time is quite plausible.

We have also considered the possible emission of hot LO phonons by holes in quantum wells. The excess energy of photoexcited heavy holes is too small to generate an appreciable number of LO phonons. The number of photoexcited light holes is much smaller than the number of electrons scattered into the $L$ and $X$ valleys within 1 ps. Thus the most likely source of hot phonons observed in our experiment is the intravalley relaxation of hot electrons in the L valleys.

\section{CONCLUSION}

In conclusion, we have studied hot carrier relaxation in GaAs/AlAs quantum wells by subpicosecond Raman scattering as a function of well thickness and carrier density. We found that electron temperatures in quantum wells were almost the same as in bulk GaAs. However, there is a dramatic decrease in the hot LO phonon population observed in Raman scattering as the well thickness is decreased. Due to confinements of electrons in the $\Gamma$ valley and of $L O$ phonons in quantum wells, the hot LO phonons emitted during the relaxation of the $\Gamma$ valley electrons cannot be observed by Raman scattering in quantum wells with $L<j 00$ A. This suppresion of hot LO phonons generated by the $\Gamma$ valley electrons has made it possible to observe larger wavevector hot $L O$ phonons generated ty other mechanisms. We found that the hot $L O$ phonon population observed in samples with $L=120$ and $230 \AA$ can be explained by the intravalley relaxation of hot electrons in the $\mathrm{L}$ valleys.

\section{ACKNOWLEDGEMENTS}

We are grateful to Hyunchul Sohn and Professor Shyh Wang of the Department of Electrical Engineering and Computer Science at Berkeley and Dr. K. T. Chan of HewlettPackard Co., Santa Rosa, California for kindly providing us with the samples used in this experiment. This work was supported by the Director, Office of Energy Research, Office of Basic Energy Science, Materials Science Division of the U.S. Deparment of Energy under Contract No. DE.AC03-76SF00098. 


\section{REFERENCES}

1. D.S. Kim and F.Y. Yu, Phys. Rev. Lett. 64946 (1990)

2. M.C. Tatham, J.F. Ryan, and C.T. Foxon, Phys.Rev.Lett. 63, 1637 (1989)

3. J. Feldmann, R. Satimann, E.O. Göbel, J. Kuhl, J. Hebling, K. Ploog, R. Muralidharan, P. Dawson, and C.T. Foxon, Phys. Rev. Lett. 62, 1892 (1989)

4. P. Saeta, J.F. Federici, R.J. Fischer, B.I. Greene, L. Pfeiffer, R.C. Spitzer, and B.A. Wilson, Appl. Fhys. Lett. 54, 1681 (1989)

5. W.H. Knox, D.S. Chemla, D.A.B. Milter, J.B. Stark, and S. Schimitt-Rink, Phys. Rev. Lett. 62, 1189 (1989)

6. R. Haight and J.A. Silbermann, Phys. Rev. Lett. 62, 815 (1989)

7. P.C. Becksr, H.L. Fragnito, C.H. Brito Cruz, R.L. Fork, J.E. Cunningham, J.E. Henry, and C. Y. Shank, Phys. Rev. Lett. 61, 1647 (1988)

8. J. Shah, B. Deveaud, T.C. Damen, W.T. Tsang, and P. Lugli, Phys. Rev. Lett. 59, 2222 (1987)

9. B.K. Ridley, Phys. Rev. B 39, 5282 (1989)

10. A.K. Sood, J. Menendez, M. Cardona, and K. Ploog, Phys. Rev. Lett. 54, 2111 (1985)

11. M.Y. Klein, IEEE J. Quantum Electron. OE-22, 1760 (1986)

12. J.K. Jain and S. Das Sarma, Phys. Rev. Lett. 62, 2305 (1989)

13. D.S. Kim and P.Y. Yu (to be published in Appl. Phys. Lett. $\$ 6,1990$ )

14. C.L. Collins and P.Y. Yu, Phys. Rev. B 30, 4501 (1984)

15. C.L. Collins and P.Y. Yu, Phys. Rev. B 27, 2602 (1983)

16. M.V. Klein in Light Scatcering in Solids. Topics in Apolied Physics, Vol. 8, ed. by M. Cardona (Springer-Verlag, New York, 1975) p. 148

17. Y. H. Huang and P.Y. Yu, Solid State Commun. 63, 109 (1987) 